\title{
The Efficacy of Bioagents against Post Flowering Stalk Rot (PFSR) of Specialty Corn Caused by Fusarium verticillioides (sheldon)
}

\author{
Anita Jat ${ }^{1}$, S. S. Sharma ${ }^{2}$ and Hansraj Dhakar ${ }^{3}$
}

Dept. of Plant Pathology, Rajasthan College of Agriculture, Maharana Pratap University of Agriculture and Technology, Udaipur (313 001), Rajasthan

\section{Corresponding Author}

Anita Jat

e-mail: anitajat670@gmail.com

\author{
Article History \\ Article ID: 3 C0536 \\ Received in $20^{\text {th }}$ October, 2017 \\ Received in revised form $25^{\text {th }}$ December, 2017 \\ Accepted in final form $19^{\text {th }}$ January, 2018
}

\begin{abstract}
The efficacy of three bio-agents viz. Pratap Trichoderma viride-1, Trichoderma viride and Trichoderma harzianum were assessed in vitro against four isolates (Fv SC-01, Fv SC- 02, Fv SC-03 and Fv SC- 04) of Fusarium verticillioides, cause Post flowering stalk rot (PFSR) of specialty corn. All used bio- agents were antagonistic in nature and significantly inhibited the growth of Fusarium verticillioides. Among the bioagents tested maximum inhibition of mycelium growth was observed by Pratap Trichoderma viride-1 @ 1500 ppm on Fv SC-04. Out of these antagonists, the resident isolate Pratap Trichoderma viride -1(78.45\%) provided the highest inhibitory effect followed by Trichoderma viride (78.22\%) and Trichoderma harzianum (56.60\%) was the lowest suppressive antagonist. Among all isolates of Fusarium verticillioides Fv SC-04 was maximum inhibited by all tested bio- agents.
\end{abstract}

Keywords: Fusarium verticillioides, Trichoderma, in vitro and Specialty Corn

\section{Introduction}

Maize (Zea mays L.) is monocotyledonous plant that belongs to grass family (Poaceae). The specialty corn viz. Pop corn, Baby corn, Sweet corn and QPM are increasing its hectares because of high economic returns. QPM and sweet corn having opaque-2 and Shrunken-2 gene respectively (Jat et al., 2009). Trichoderma spp. are fungi that are present in nearly all soils, they frequently are the most prevalent cultivable fungi (Ubalua and Oti, 2007). They are common inhitants of the rhizosphere and are well recognized as biological agents of soil borne plant pathogens. For controlling the disease, farmers usually applied synthetic fungicides to the plants. However, these treatments were ineffective because it led to the appearance of environmental contaminations (Jacobsen and Backman, 1993). Hence, biological control using microbial agents is an alternative way to manage plant pathogens because it's eco-friendly and cost-effective. Nowadays, many microbes have been used as biocontrol agent of plant disease including Trichoderma spp. The use of Trichoderma spp. as a biocontrol agent against Fusarium spp. showed a high efficacy when tested under in vitro and in vivo conditions (Thangavelu et al., 2004). Therefore, the purpose of this study was to screen the pathogens of PFSR and antagonistic ability of Trichoderma spp. to suppress the growth of PFSR pathogen under in vitro conditions.

\section{Materials and Methods}

The experiment was conducted at Department of Plant Pathology, Rajasthan College of Agriculture (RCA) MPUAT, Udaipur during kharif, 2015-16.

\subsection{Isolation of biocontrol agents}

Isolation of biocontrol agent (Trichoderma spp.) was attempted from air dried rhizosphere soil in the viscinity of healthy roots of specialty corn. Specific technique was employed for isolation of resident (native) biocontrol agent. The details of these techniques are given below.

\subsection{Isolation by baiting}

A loop full of the dried rhizosphere soil collected from specialty corn field was placed in the bottom of the empty sterilized Petri-plates and to this a drop of sterile water was added, thoroughly mixed and allowed to dry on the laminar flow bench for 3 minutes, over this, $20 \mathrm{ml}$ of lukewarm media 0.1 per cent malt extract agar (MEA) was poured carefully without disturbing the soil at the bottom. The medium in these plates was allowed to solidify and then $2 \mathrm{~mm}$ bit of growing cultures of isolated pathogen was aseptically inoculated in each plate. The plates were incubated at $25 \pm 2{ }^{\circ} \mathrm{C}$ and examined after 2 days for growth of the organism under stereo binocular microscope and hyphae of antagonists which appeared piercing through the hyphae of the pathogens or found 
growing over the pathogens or causing lysis thereof, were aseptically picked up with the help of sterilized needle and transferred on to fresh MEA plates and purified with hyphal tip culture technique and maintained in MEA slants.

\section{Results and Discussion}

Efficacy of bioagents of local isolates and procured cultures of Trichoderma spp. were studied in vitro as described in Material and Methods. Data revealed that Trichoderma spp. were potential antagonists against $F$. verticillioides. The resident isolate which was found fast growing and effective against pathogen $F$. verticillioides was identified as Trichoderma viride based on morphological characters and was designated as Pratap Trichoderma viride-1 (PTV-1) this isolates has to be registered with ITCC for accession number. All the tested biocontrol agents significantly $(p=0.05)$ inhibited the mycelial growth at all concentrations from 500, 1000 and 1500 ppm.

Pratap Trichoderma viride -1 caused 55.42, 56.05, 56.45 and $56.77 \%$ inhibition of mycelium growth at $500 \mathrm{ppm}$ concentration, at $1000 \mathrm{ppm}$ it caused 64.15, 65.37, 66.45 and $66.77 \%$ inhibition of mycelium growth and $77.55,77.87$, 78.10 and $78.45 \%$ inhibition of mycelium growth at 1500 ppm in (Fv SC-01, 02, 03 and 04) all isolates respectively. The maximum per cent inhibition of mycelium growth i.e. $78.45 \%$ of $F$. verticillioides SC-04 isolate was followed by Fv SC-03 that caused $78.10 \%$ inhibition of mycelial growth.

Trichoderma viride caused 47.65, 49.87, 49.55 and $50.85 \%$ inhibition of mycelium growth at 500 ppm concentration, at 1000 ppm it caused $61.95,63.11,63.33$ and $63.77 \%$ inhibition of mycelium growth and 75.44, 76.22, 76.52 and $78.22 \%$ inhibition of mycelium growth at 1500 ppm of (Fv SC-01, 02, 03 and 04) all isolates respectively. The maximum per cent inhibition of mycelium growth i.e. $78.22 \%$ of $F$. verticillioides SC-04 isolate was followed by Fv SC-03 that caused $76.52 \%$ inhibition of mycelial growth.

Trichoderma harzianum was slightly less effective, as it caused $26.85,27.72,27.85$ and $28.72 \%$ inhibition of mycelial growth at 500 ppm concentration, at 1000 ppm it caused 39.72, 41.95, 43.22 and $45.55 \%$ inhibition of growth and 54.37, 54.95, 55.66 and $56.60 \%$ inhibition of mycelium growth at $1500 \mathrm{ppm}$ in (Fv SC-01, 02, 03 and 04) all isolates respectively. The maximum per cent inhibition of mycelium growth i.e. 56.60 per cent of F. verticillioides SC-04 isolate was followed by Fv SC-03 that caused 55.66\% inhibition of mycelial growth. (Table 1, 2, 3, 4).

Suhaida and Izzati, 2013 based on virulence assay, $F$. proliferatum $\mathrm{B} 202 \mathrm{C}$ was the most pathogenic isolate among other species including $F$. verticillioides. This pathogen was challenged in dual culture assays with 72 isolates of Trichoderma spp., which were isolated from soil samples. T. harzianum T73s showed highest per centage inhibition of 73.10\% was further tested for its efficacy to suppress FER under glasshouse conditions. Ferrigo et al., 2014 reported that the ability of the biocontrol fungus Trichoderma harzianum to
Table 1: Antagonistic activity (Per cent inhibition) of two isolates Trichoderma viride and one isolate of $T$. harzianum against Fusarium verticillioides SC-01 isolate in vitro

\begin{tabular}{|c|c|c|c|c|c|c|c|}
\hline \multirow[t]{2}{*}{$\begin{array}{l}\text { SI. } \\
\text { No. }\end{array}$} & \multirow[t]{2}{*}{ Con } & \multicolumn{3}{|c|}{$\begin{array}{l}\text { Isolates of Tricho- } \\
\text { derma spp. Growth* } \\
\text { Mycelial growth } \\
\text { (mm) }\end{array}$} & \multicolumn{3}{|c|}{$\begin{array}{l}\text { Per cent growth inhi- } \\
\text { bition }^{*}\end{array}$} \\
\hline & & PT & TV & $\mathrm{TH}$ & PT & TV & $\mathrm{TH}$ \\
\hline 1. & 500 & 40.07 & 47.05 & 65.80 & $\begin{array}{c}55.42 \\
(48.09)\end{array}$ & $\begin{array}{c}47.65 \\
(43.63)\end{array}$ & $\begin{array}{c}26.85 \\
(31.19)\end{array}$ \\
\hline 2. & 1000 & 32.25 & 34.20 & 54.20 & $\begin{array}{c}64.15 \\
(53.19)\end{array}$ & $\begin{array}{l}61.95 \\
51.89)\end{array}$ & $\begin{array}{c}39.72 \\
(39.05)\end{array}$ \\
\hline 3. & 1500 & 20.15 & 22.10 & 41.02 & $\begin{array}{c}77.55 \\
(61.69)\end{array}$ & $\begin{array}{l}75.44 \\
60.24)\end{array}$ & $\begin{array}{c}54.37 \\
(47.49)\end{array}$ \\
\hline \multirow[t]{2}{*}{4.} & $\begin{array}{l}\text { Con- } \\
\text { trol }\end{array}$ & 90.00 & 90.00 & 90.00 & $\begin{array}{l}00.00 \\
(0.0)\end{array}$ & $\begin{array}{c}0.0 \\
(0.0)\end{array}$ & $\begin{array}{c}0.0 \\
(0.0)\end{array}$ \\
\hline & & SEm \pm & \multicolumn{2}{|c|}{$\operatorname{CD}(p=0.05)$} & SEm+ & \multicolumn{2}{|c|}{$\mathrm{CD}(p=0.05)$} \\
\hline \multicolumn{2}{|c|}{ Biocontrol } & 0.04 & \multicolumn{2}{|c|}{0.119} & 0.06 & \multicolumn{2}{|c|}{0.133} \\
\hline \multicolumn{2}{|c|}{$\begin{array}{l}\text { Concen- } \\
\text { tration }\end{array}$} & 0.04 & \multicolumn{2}{|c|}{0.119} & 0.05 & \multicolumn{2}{|c|}{0.133} \\
\hline \multicolumn{2}{|c|}{$\mathrm{B} \times \mathrm{C}$} & 0.07 & \multicolumn{2}{|c|}{0.207} & 0.08 & \multicolumn{2}{|c|}{0.230} \\
\hline
\end{tabular}

Con: Concentration (ppm); PT: Pratap T. viride -1; TV: T. viride; TH: T. harzianum; *Mean of four replications; Figures in parentheses are arcsine $\vee$ per cent angular transformed values

Table 2: Antagonistic activity (Per cent inhibition) of two isolates Trichoderma viride and one isolate of T. harzianum against Fusarium verticillioides SC-02 isolate in vitro

\begin{tabular}{|c|c|c|c|c|c|c|c|}
\hline \multirow[t]{2}{*}{$\begin{array}{l}\text { SI. } \\
\text { No. }\end{array}$} & \multirow[t]{2}{*}{ Con } & \multicolumn{3}{|c|}{$\begin{array}{l}\text { Isolates of Tricho- } \\
\text { derma spp. Growth* } \\
\text { Mycelial growth } \\
\text { (mm) }\end{array}$} & \multicolumn{3}{|c|}{$\begin{array}{l}\text { Per cent growth inhi- } \\
\text { bition }^{*}\end{array}$} \\
\hline & & PT & TV & $\mathrm{TH}$ & PT & TV & $\mathrm{TH}$ \\
\hline 1. & 500 & 39.5 & 45.10 & 65.00 & $\begin{array}{l}56.05 \\
(48.45)\end{array}$ & $\begin{array}{c}49.87 \\
(44.47)\end{array}$ & $\begin{array}{c}27.72 \\
(31.75)\end{array}$ \\
\hline 2. & 1000 & 31.12 & 33.20 & 52.20 & $\begin{array}{c}65.37 \\
(53.93)\end{array}$ & $\begin{array}{c}63.11 \\
(52.57)\end{array}$ & $\begin{array}{c}41.95 \\
(40.85)\end{array}$ \\
\hline 3. & 1500 & 19.87 & 21.40 & 40.00 & $\begin{array}{c}77.87 \\
(61.91)\end{array}$ & $\begin{array}{c}76.22 \\
(60.77)\end{array}$ & $\begin{array}{c}54.95 \\
(47.42)\end{array}$ \\
\hline \multirow[t]{2}{*}{4.} & $\begin{array}{l}\text { Con- } \\
\text { trol }\end{array}$ & 90.00 & 90.00 & 90.00 & $\begin{array}{l}0.0 \\
(0.0)\end{array}$ & $\begin{array}{c}0.0 \\
(0.0)\end{array}$ & $\begin{array}{c}0.0 \\
(0.0)\end{array}$ \\
\hline & & SEm \pm & \multicolumn{2}{|c|}{$\operatorname{CD}(p=0.05)$} & SEm+ & \multicolumn{2}{|c|}{$\operatorname{CD}(p=0.05)$} \\
\hline \multicolumn{2}{|c|}{ Biocontrol } & 0.03 & \multicolumn{2}{|c|}{0.111} & 0.04 & \multicolumn{2}{|c|}{0.127} \\
\hline \multicolumn{2}{|c|}{$\begin{array}{l}\text { Concen- } \\
\text { tration }\end{array}$} & 0.03 & \multicolumn{2}{|c|}{0.111} & 0.04 & \multicolumn{2}{|c|}{0.127} \\
\hline \multicolumn{2}{|c|}{$\mathrm{B} \times \mathrm{C}$} & 0.07 & \multicolumn{2}{|c|}{0.193} & 0.08 & \multicolumn{2}{|c|}{0.220} \\
\hline
\end{tabular}

*Mean of four replications; Figures in parentheses are arcsine $V$ per cent angular transformed values 
Table 3: Antagonistic activity (Per cent inhibition) of two isolates Trichoderma viride and one isolate of $T$. harzianum against Fusarium verticillioides SC-03 isolate in vitro

\begin{tabular}{|c|c|c|c|c|c|c|c|}
\hline \multirow[t]{2}{*}{$\begin{array}{l}\text { SI. } \\
\text { No. }\end{array}$} & \multirow[t]{2}{*}{ Con } & \multicolumn{3}{|c|}{$\begin{array}{l}\text { Isolates of Tricho- } \\
\text { derma spp. Growth* } \\
\text { Mycelial growth } \\
(\mathrm{mm})\end{array}$} & \multicolumn{3}{|c|}{$\begin{array}{l}\text { Per cent growth inhibi- } \\
\text { tion }^{*}\end{array}$} \\
\hline & & PT & TV & TH & PT & TV & TH \\
\hline 1. & 500 & 39.15 & 45.20 & 64.90 & $\begin{array}{l}56.45 \\
(48.68)\end{array}$ & $\begin{array}{l}49.55 \\
(44.72)\end{array}$ & $\begin{array}{r}27.85 \\
(31.83)\end{array}$ \\
\hline 2. & 1000 & 30.15 & 33.00 & 51.10 & $\begin{array}{c}66.45 \\
(54.58)\end{array}$ & $\begin{array}{c}63.33 \\
(52.69)\end{array}$ & $\begin{array}{l}43.22 \\
(41.07)\end{array}$ \\
\hline 3. & 1500 & 19.67 & 21.10 & 39.90 & $\begin{array}{l}78.10 \\
(62.07)\end{array}$ & $\begin{array}{c}76.52 \\
(60.99)\end{array}$ & $\begin{array}{l}55.66 \\
(48.19)\end{array}$ \\
\hline \multirow[t]{2}{*}{4.} & $\begin{array}{l}\text { Con- } \\
\text { trol }\end{array}$ & 90.00 & 90.00 & 90.00 & $\begin{array}{c}0.0 \\
(0.0)\end{array}$ & $\begin{array}{c}0.0 \\
(0.0)\end{array}$ & $\begin{array}{l}0.0 \\
(0.0)\end{array}$ \\
\hline & & SEm \pm & \multicolumn{2}{|c|}{$\mathrm{CD}(p=0.05)$} & SEm+ & \multicolumn{2}{|c|}{$C D(p=0.05)$} \\
\hline \multicolumn{2}{|c|}{ Biocontrol } & 0.04 & \multicolumn{2}{|c|}{0.124} & 0.05 & \multicolumn{2}{|c|}{0.131} \\
\hline \multicolumn{2}{|c|}{$\begin{array}{l}\text { Concentra- } \\
\text { tion }\end{array}$} & 0.04 & \multicolumn{2}{|c|}{0.124} & 0.05 & \multicolumn{2}{|c|}{0.131} \\
\hline \multicolumn{2}{|c|}{$B \times C$} & 0.07 & \multicolumn{2}{|c|}{0.216} & 0.08 & \multicolumn{2}{|c|}{0.228} \\
\hline
\end{tabular}

*Mean of four replications; Figures in parentheses are arcsine $\checkmark$ per cent angular transformed values

Table 4: Antagonistic activity (Per cent inhibition) of two isolates Trichoderma viride and one isolate of T. harzianum against Fusarium verticillioides SC-04 isolate in vitro

SI. Con Isolates of Tricho- Per cent growth inhibiNo. derma spp. Growth* tion* Mycelial growth

\begin{tabular}{|c|c|c|c|c|c|c|c|}
\hline & \multicolumn{3}{|c|}{$(\mathrm{mm})$} & \multirow[b]{2}{*}{ PT } & \multirow[b]{2}{*}{ TV } & \multirow[b]{2}{*}{$\mathrm{TH}$} \\
\hline & & PT & TV & $T H$ & & & \\
\hline 1. & 500 & 38.85 & 44.20 & 64.10 & $\begin{array}{c}56.77 \\
(48.87)\end{array}$ & $\begin{array}{c}50.85 \\
(45.46)\end{array}$ & $\begin{array}{c}28.72 \\
(32.39)\end{array}$ \\
\hline 2. & 1000 & 29.85 & 32.57 & 49.00 & $\begin{array}{c}66.77 \\
(54.77)\end{array}$ & $\begin{array}{c}63.77 \\
(52.92)\end{array}$ & $\begin{array}{c}45.55 \\
(42.40)\end{array}$ \\
\hline 3. & 1500 & 19.15 & 19.60 & 39.10 & $\begin{array}{c}78.45 \\
(62.31)\end{array}$ & $\begin{array}{c}78.22 \\
(62.14)\end{array}$ & $\begin{array}{c}56.60 \\
(48.71)\end{array}$ \\
\hline \multirow[t]{2}{*}{4.} & $\begin{array}{l}\text { Con- } \\
\text { trol }\end{array}$ & 90.00 & 90.00 & 90.00 & $\begin{array}{c}0.0 \\
(0.0)\end{array}$ & $\begin{array}{c}0.0 \\
(0.0)\end{array}$ & $\begin{array}{c}0.0 \\
(0.0)\end{array}$ \\
\hline & & SEm \pm & \multicolumn{2}{|c|}{$C D(p=0.05)$} & SEm+ & \multicolumn{2}{|c|}{$\mathrm{CD}(p=0.05)$} \\
\hline \multicolumn{2}{|c|}{ Biocontrol } & 0.03 & \multicolumn{2}{|c|}{0.103} & 0.04 & \multicolumn{2}{|c|}{0.122} \\
\hline \multicolumn{2}{|c|}{$\begin{array}{l}\text { Concentra- } \\
\text { tion }\end{array}$} & 0.03 & \multicolumn{2}{|c|}{0.103} & 0.04 & \multicolumn{2}{|c|}{0.122} \\
\hline \multicolumn{2}{|c|}{$B \times C$} & 0.06 & \multicolumn{2}{|c|}{0.179} & 0.07 & \multicolumn{2}{|c|}{0.212} \\
\hline
\end{tabular}

*Mean of four replications; Figures in parentheses are arcsine $\checkmark$ per cent angular transformed values induce systemic resistance in maize against $F$. verticillioides is still lacking. Shekhar and Kumar, 2010 tested efficacy of six isolates of $T$. harzianum using dual culture plate technique and inhibition through volatile substances against charcoal rot pathogen of maize and found that Hyderabad isolate of $T$. harzianum caused maximum inhibition (62.3\%) of radial growth of Macrophomina phaseolina and regarded as potential bio-control agent for minimizing.

\section{Conclusion}

To develop effective management strategies for PFSR three bio-control agents were evaluated against $F$. verticillioides (Fv SC-01 to Fv SC-04) in vitro condition. Pratap Trichoderma viride-1@ 500, 1000 and 1500 ppm was found most effective in inhibiting the mycelial growth of pathogen. Among the bioagents tested maximum inhibition of mycelium growth was observed by Pratap Trichoderma viride-1 @ 1500 ppm on Fv SC-04.

\section{Acknowledgement}

I would like to thank the Plant Pathology Research farm and Department of Plant Pathology, MPUAT, Udaipur, Rajasthan for providing all possible research facilities while executing the field experiment and laboratory analysis.

\section{References}

Ferrigo, D., Raiola, A., Piccolo, E., Scopel, C. and Causin, R. 2014. Trichoderma harzianum t22 induces in maize systemic resistance against Fusarium verticillioides. Journal of Plant Pathology 96, 133-142.

Jacobsen, B.J., Backman, P.A., 1993. Biological and cultural plant disease control: Alternative and supplements to chemical in IPM systems. Plant Disease 77, 311-315

Jat, M.L., Dass, S., Yadav, V.K., Sekhar and Singh, D.K., 2009. Quality protein maize for food and nutritional security in India.

Shekhar, M., Kumar, S., Sharma, R.C., Singh, R., 2010. Sources of resistance against post- flowering stalk rots of Maize. Archives Phytopathology Plant Protection 43, 259-263.

Suhaida, S., Nur Ainlzzati, M.Z., 2013. The efficacy of Trichoderma harzianum T73s as a biocontrol agent of Fusarium ear rot disease of maize. International Journal Agriculture and Biology 15, 1175-1180.

Thangavelu, R., Palaniswami, A., Velazhahan, R., 2004. Mass production of Trichoderma harzianum for managing Fusarium wilt of banana. Agriculture, Ecosystems and Environment. 103, 259-263.

Ubalua A. O.,Oti C.,2007. Antagonistic properties of Trichoderma viride on Post-harvest cassva root pathogen. African Journal of Biotechnology 6, 24472450. 